\title{
Biaxiality-induced magnetic field effects in bent-core nematics: molecular field and Landau theory
}

\author{
T.B.T. To, ${ }^{1}$ T.J. Sluckin, ${ }^{1, *}$ and G.R. Luckhurst ${ }^{2}$ \\ ${ }^{1}$ School of Mathematics, University of Southampton, Southampton, SO17 1BJ, United Kingdom \\ ${ }^{2}$ School of Chemistry, University of Southampton, Southampton, SO17 1BJ, United Kingdom
}

(Dated: June 12, 2013)

\begin{abstract}
Nematic liquid crystals composed of bent-core molecules exhibit unusual properties, including an enhanced Cotton-Mouton effect and an increasing isotropic (paranematic)-nematic phase transition temperature as a function of magnetic field. These systems are thought to be good candidate biaxial liquid crystals. Prompted by these experiments, we investigate theoretically the effect of molecular biaxiality on magnetic field-induced phenomena for nematic liquid crystals, using both molecular field and Landau theory. The geometric mean approximation is used in order to specify the degree of molecular biaxiality using a single parameter. We reproduce experimental field-induced phenomena, and predict also an experimentally accessible magnetic critical point. The Cotton-Mouton effect and temperature dependence of the paranematic-nematic phase transition are more pronounced with increased molecular biaxiality. We compare our theoretical approaches and make contact with recent relevant experimental results on bent-core molecular systems.
\end{abstract}

PACS numbers: $78.15 .+\mathrm{e}, 64.70 . \mathrm{M}-$.

\section{INTRODUCTION}

In external fields, nematic-forming fluids exhibit a number of common characteristics close to the onset of the nematic phase [1]. First, the field induces a low degree of orientational order in the isotropic phase, causing it now to be known as a paranematic phase. The order parameter $\left\langle P_{2}\right\rangle$ of the new phase is proportional to the quadratic power of the applied field. This phenomenon is called the Cotton-Mouton effect if the field is magnetic and the Kerr effect if the field is electric. Second, the temperature $T_{N P}$ of the first order phase transition from the more ordered phase to the less ordered phase is shifted towards a higher temperature as the field strength increases. This shift in the transition temperature also increases linearly with the quadratic power of the applied field strength. Finally, there is a critical field strength, above which it is predicted that there will be no clear transition between the nematic and the paranematic phases.

Early experimental studies by Stinson and Litster using just a magnetic field revealed a Cotton-Mouton effect [2] in nematics formed of rod-like (or calamitic) molecules, but did not observe a temperature shift in $T_{N P}$. Later Rosenblatt [3] was able to observe an increase in the transition temperature, but the shift was negligible, of only a few $\mathrm{mK}$. More recently, all three fieldinduced phenomena have been observed experimentally for a system of calamitic molecules in electric fields by Lelidis and Durand [4]. However, in general these effects are also weak and difficult to observe. In recent years analogous experiments have been carried out on systems of bent-core molecules. Wiant et al.[5] observed a Cotton-

* Corresponding author: T.J.Sluckin@soton.ac.uk
Mouton effect, while in related work Ostapenko et al. [6], using the bent-core molecule 4-chlororesorcinol bis[4-(4n-dodecyloxybenzoyloxy) benzoate] (ClPbis10bbs) (see Fig.1 observed both a strong Cotton-Mouton effect and a marked increase in $T_{N P}$. The implication is that in bent-core nematics, magnetic effects on the nematicparanematic phase transition may be stronger than in conventional calamitic nematics, and the consequent magnetic critical point may also be much more accessible. This paper addresses the theoretical question of why this might be the case.

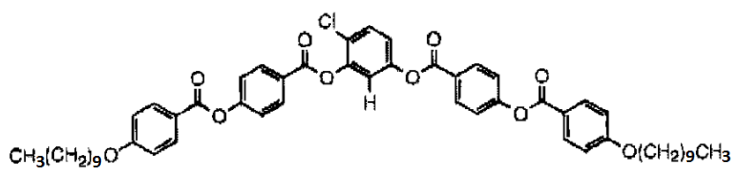

FIG. 1. The molecular structure of the liquid crystal material 4- chlororesorcinol bis[4-(4-n-dodecyloxybenzoyloxy) benzoate] (ClPbis10bbs) used in the experiment by Ostapenko et $a l .[6]$.

The effect of an external field in general on the nematic-isotropic phase transition can be addressed using either the Landau-de Gennes theory, or by using molecular field theory. However, the question of the origin of the field (electric, magnetic etc.) makes little difference to the mathematical structure of the theory. Landau-de Gennes theories for electric [4] and magnetic $[1,7]$ fields both give analytical formulas for the three field-induced phenomena discussed above. Analogous calculations for nematics formed from calamitic molecules, using Maier-Saupe-like molecular field theories in the presence of an external field, have been made by Hanus [8] (electric fields) and Wojtowicz and Sheng [9] (magnetic fields). A comparison of the Landau-de Gennes theory and the analogous molecular field theory 
for such a magnetic field-induced nematic was carried out by Palffy-Muhoray and Dunmur [10]. In positive diamagnetic anisotropy materials, both paranematic and nematic align with the director parallel to the field. However, for negative anisotropy materials, the director tends to align perpendicular to the field in the nematic phase, but parallel to it in the paranematic phase.

This general picture has also been confirmed by simulation. Luckhurst and Simpson [11] carried out a series of Lebwohl-Lasher [12] lattice Monte Carlo simulations for a nematic in a magnetic field, which supported the hypothesis of a critical field strength. A more recent series of lattice simulations, using the Zwanzig model of discrete orientations [13], has been performed by by Warsono et al.[14]. This simple simulation also reproduces the magnetic field-induced nematic-to-paranematic phase transition and the critical field.

Ostapenko et al [6] associated the strong effects which they observed in their experimental system with two primary factors. The first was the strong magnetic field $(\sim$ $31 \mathrm{~T}$ ) available. The second was that the material under study was formed from bent-core molecules. Within the Landau-de Gennes theory, a strong Cotton-Mouton effect at $T_{N I}$ and a strong shift in $T_{N P}(B)$ would occur if the coefficient of the cubic term in the Landau-de Gennes expansion were in some sense small. They argue empirically for a connection between the smallness of the cubic parameter and the bent-core $\mathrm{V}$-shape. In this paper, we shall specifically address the question of the connection between these quantities, noting that the constituent bent-core molecules used in this experiment are highly biaxial.

This bulk of this paper is concerned with the effect of molecular biaxiality on magnetic-field induced phenomena for nematic liquid crystals, with an underlying goal of addressing the recent experimental findings [6] on bent-core molecule liquid crystals discussed above. The high biaxiality of these molecules depends on the interarm angle. As a result, we expect the magnitudes of the magnetic-field phenomena for bent-core molecule systems to be different from those in calamitic molecule systems. We develop a molecular field theory as an extension of the theory by Wojtowicz and Sheng [9] since it is a straightforward theory which allows us to include molecular biaxiality. We note that a simple molecular field theory for uniaxial nematic formed from biaxial molecules in the absence of any external field was developed some time ago by Luckhurst et al.[15]. This theory has been used to improve the numerical agreement between theory and experiment of the major order parameter $S$ at the transition, the transitional entropy, the dependence of $S$ on the temperature and the non-zero value of the molecular biaxial order parameter $D$ [15]. In this context, the present theory may also be viewed as an extension of that theory for a uniaxial nematic formed from biaxial molecules, now also specifically including the interaction of the constituent molecules with the magnetic field. The layout of the paper is as follows.
The molecular field theory is discussed in section II. In addition to studying the molecular field theory, we also derive a Landau-de Gennes expansion from the molecular field theory for uniaxial nematic formed from biaxial molecules in section III. The methodology is an extension of that developed by Katriel et al.[16] for uniaxial nematic formed from uniaxial molecules, and later extended by Luckhurst et al.[17] to study nematics formed from biaxial molecules. Using this method, we demonstrate that the cubic expansion coefficient $\mathcal{B}$ can be related to the molecular biaxiality. Since typical bent-core molecules are highly biaxial and depend on the angle between the two arms, their system has a significantly smaller value of $\mathcal{B}$ than for calamitic molecules. Finally, we discuss our results in section IV.

\section{MOLECULAR FIELD THEORY}

\section{A. Self-consistent free energy}

a. Background to theory. The theory combines elements of the molecular field theory of biaxial liquid crystals developed elsewhere [15, 18-21], with the molecular field theory of uniaxial liquid crystals in an external field [9]. Molecular orientation $\omega$ is parameterized in terms of two Euler angles $\beta, \gamma$ taking their conventional meanings (polar angle $\beta$ and azimuthal angle $\gamma$ ). The Euler angle $\alpha$ is only of interest in a specifically biaxial phase, and is not required here. In this part of the theory, we suppose constituent molecules to possess $D_{2 h}$ symmetry (i.e that of an orthogonal parallellepiped). We note, however, that the molecules of ultimate interest in this theory possess $C_{2 v}$ symmetry. Nevertheless, the second order invariants associated with these two different symmetries are the same.

b. Spherical harmonics. Two second-order spherical harmonics play an important role in a uniaxial nematic formed from biaxial molecules. These are:

$$
\begin{gathered}
C_{20}(\omega)=\frac{1}{2}\left(3 \cos ^{2} \beta-1\right)=P_{2}(\cos \beta), \\
\operatorname{Re} C_{22}(\omega)=\sqrt{\frac{3}{8}} \sin ^{2} \beta \cos 2 \gamma,
\end{gathered}
$$

where $P_{2}(\cos \beta)$ is the usual second order Legendre polynomial. The required order parameters are the mean values of these quantities:

$$
\begin{gathered}
S=\left\langle C_{20}(\omega)\right\rangle=\left\langle\frac{1}{2}\left(3 \cos ^{2} \beta-1\right)\right\rangle=\bar{P}_{2}, \\
D=\left\langle\operatorname{Re} C_{22}(\omega)\right\rangle
\end{gathered}
$$

The order parameter $D$ gives information about the relative degrees of orientation of the minor order axes, i.e. its biaxiality. A detailed discussion of order parameters and their significance, as well as conventions in theories of biaxial liquid crystals, is given by Rosso [22] 
c. Biaxiality parameter and geometric-mean approximation. We now formulate the internal energy per molecule. In zero field, this internal energy is constructed as a rotational invariant of the quadratic products of the second-rank order parameters [20],

$$
U_{M}=-\frac{1}{2} \sum u_{2 m n}\left\langle C_{2 m}\right\rangle\left\langle C_{2 n}\right\rangle \text {. }
$$

Here, the scalar components of the supertensor $u_{2 m n}$ are called interaction coefficients. Since the constituent molecules have $C_{2 v}$ symmetry, there are only three second-rank interaction coefficients, $u_{200}, u_{202} \equiv u_{220}$ and $u_{222}[23]$.

In order to reduce the number of parameters, we use the geometric-mean approximation [15] (GMA). In this, the molecular interaction only depends on one relative biaxiality parameter, $\lambda=u_{222} / u_{202}=u_{202} / u_{200}$. We note that the notation $\lambda$ for the biaxiality parameter is consistent with the usage of Luckhurst et al.[15], but differs from that of Sonnet et al. [24], and of Luckhurst et al.[17]. A key simplification of the GMA, at least within molecular field theory, is that now there is only one combination of spherical harmonics $J$ entering the theory $[15,17]$. Likewise there is only a single independent order parameter $\mathcal{J}=\langle J(\omega)\rangle$ (the composite order parameter), where

$$
J(\omega)=C_{20}(\omega)+2 \lambda C_{22}(\omega) .
$$

In the presence of a magnetic field, the internal energy consists of two parts. The first part is the molecular interaction, $U_{M}$. The second part, $U_{B}$, is generated by the interaction of the molecule with the applied field. Following de Gennes [25], this is given by

$$
U_{B}=-\frac{3}{4} B^{2} \sum_{n=0}^{2} \chi_{2 n}\left\langle C_{2 n}\right\rangle
$$

where $B$ and $\chi_{2 n}$ denote the magnetic flux density and the molecular magnetic susceptibility tensor, respectively. There are two strength parameters $\chi_{20}$ and $\chi_{22}$. An approximation in the spirit of the GMA is that

$$
\chi_{22}=\lambda \chi_{20} .
$$

The underlying assumption here is that the biaxiality in $\chi_{m n}$ is the same as in $u_{2 m n}$, which is plausible, although at this stage untested.

Subject to these approximations, the internal energy of a biaxial molecule in a uniaxial nematic in the presence of a magnetic field reduces to

$$
\begin{aligned}
U=U_{M}+U_{B}= & -\frac{1}{2} u_{200} \mathcal{J}^{2}-\frac{3}{4} \chi_{20} B^{2} \mathcal{J} \\
& =u_{200}\left[-\frac{1}{2} \mathcal{J}^{2}-V \mathcal{J}\right],
\end{aligned}
$$

which is a function only of the single composite order parameter $\mathcal{J}$. It is apparent that the elimination of the second independent parameter simplifies the mathematical problem significantly. The term on the extreme right scales all energies with respect to $u_{200}$. The quantity

$$
V=\frac{3}{4} \frac{B^{2} \chi_{20}}{u_{200}}
$$

serves as a non-dimensional measure of the effective imposed external field. It is proportional to the square of the imposed magnetic or electric field, and is also proportional to the physical coupling constants. A scaled temperature can also be expressed in terms of $u_{200}$ :

$$
T^{*}=\frac{k_{B} T}{u_{200}}
$$

where $k_{B}$ is Boltzmann's constant.

d. Free energy functional. The free energy functional $A[f(\omega)]$, where $f(\omega)$ is the singlet orientational distribution function, is now constructed in the usual way [26], by combining energy and entropy terms:

$$
A=U-T \mathcal{S},
$$

where $T$ denotes the absolute temperature, and $\mathcal{S}$ is the relative orientational entropy. The order parameters can be expressed in terms of $f(\omega)$ :

$$
\left\langle C_{2 m}\right\rangle=\int d \omega C_{2 m}(\omega) f(\omega),
$$

while the composite order parameter $\mathcal{J}$ is given by:

$$
\mathcal{J}=\langle J(\omega)\rangle=\int d \omega J(\omega) f(\omega),
$$

the orientational entropy is given by

$$
\mathcal{S}=-k_{B} \int d \omega f(\omega) \ln f(\omega),
$$

and the distribution function is subject to a normalisation condition

$$
\int d \omega f(\omega)=1
$$

The distribution function $f(\omega)$ is determined by the free energy given in eqs.(7),(10)) subject to the constraint eq.(13) [25]. The resulting singlet orientational distribution function is given by

$$
f(\omega)=Q^{-1} \exp \left(U(\omega) / k_{B} T\right),
$$

where $Q$ denotes the orientational partition function

$$
Q=\int d \omega \exp \left(U(\omega) / k_{B} T\right),
$$

and where the potential of mean torque $U(\omega)$ is given by

$$
\begin{aligned}
U(\omega)=-( & \sum u_{2 m n}\left\langle C_{2 m}\right\rangle C_{2 n}(\omega) \\
& \left.+(3 / 4) B^{2} \chi_{2 n} C_{2 n}(\omega)\right) .
\end{aligned}
$$

Using eq.(7), this can be reexpressed in terms of $\mathcal{J}$ as follows:

$$
U(\omega)=-u_{200}(\mathcal{J}+V) J(\omega) .
$$


e. Self-consistent equation. Combining eqs. $(14,17)$, together with eq.(9) the quantity $\mathcal{J}$ can be expressed self-consistently in terms of the scaled temperature $T^{*}$ and scaled external field $V$ as follows:

$$
\mathcal{J}=Q^{-1} \int J(\omega) \exp (\mathcal{J}+V) J(\omega) / T^{*} d \omega
$$

with

$$
Q=\int \exp (\mathcal{J}+V) J(\omega) / T^{*} d \omega
$$

We note that evaluation of $\mathcal{J}$ is sufficient to solve for order parameters as a function of temperature, as the potential of mean torque is now specified.

f. Cotton-Mouton effect. Here we recapitulate previous work on the stability of the isotropic phase, and bifurcations rendering it unstable (see e.g. Mulder [27]). Eq.(18) can also be used to calculate the degree of order induced in the isotropic phase above $T_{N I}$. In this regime $\mathcal{J} \ll 1$, and the exponentials in eqs. $(18,19)$ can be expanded to lowest order, yielding:

$$
\mathcal{J}=\frac{1}{4 \pi} \int J(\omega)\left(1+(\mathcal{J}+V) \frac{J(\omega)}{T^{*}}\right) d \omega .
$$

The linear response of $\mathcal{J}$ to an imposed field $V$ can then formally be derived, noting that

$$
\int J(\omega) d \omega \equiv 0
$$

Thus

$$
\mathcal{J}=\frac{T_{b f}^{*}}{T^{*}}(\mathcal{J}+V)
$$

with $T_{b f}^{*}$ formally defined by

$$
T_{b f}^{*}=\frac{1}{4 \pi} \int d \omega J(\omega)^{2} .
$$

Rearanginging eq.(22), we obtain

$$
\mathcal{J}=\frac{T_{b f}^{*}}{T^{*}-T_{b f}^{*}} V
$$

in the limit that $V$ is infinitesimally small. Thus the orientational order is proportional to $V$ and hence to the square of the field, as expected, and the non-dimensional Cotton-Mouton coefficient is given by:

$$
\kappa=\left(\frac{\partial \mathcal{J}}{\partial V}\right)_{V=0}=\frac{T_{b f}^{*}}{T^{*}-T_{b f}^{*}} .
$$

The quantity $T_{b f}^{*}$ is thus seen to be the orientational spinodal temperature of the fluid in scaled units. It is the temperature at which the spontaneous response of the orientational order parameter to an orienting field would diverge in the isotropic phase. In normal circumstances, $T_{b f}^{*}<T_{N I}^{*}$, and so this temperature is not reached. But the closeness to which it is reached is dependent on the relative biaxiality $\lambda$. g. Relationship of relative biaxiality parameter to bent-core molecular shape. In this paper we discuss experiments on bent-core nematogens, characterised by an interarm angle $\theta$, while attributing their properties to biaxiality, characterised by parameter $\lambda$. This problem has been addressed by a number of authors [28, 29]; for an explicit derivation in the slightly more complex situation of different arms, see Bates and Luckhurst [30]. The basic idea is to suppose that the bent-core molecule is formed from rods glued together, supposing that the rods in different molecules act on each other using interactions with Maier-Saupe symmetry, and supposing potential energies to be additive. The key formula $[28,30]$ is:

$$
\lambda=\sqrt{\frac{3}{2}}\left(\frac{1+\cos \theta}{1-3 \cos \theta}\right) .
$$

We observe that this formula become meaningless if $\theta$ is too small, and that normally $\theta \geq \pi / 2$. The value $\theta=\pi$ corresponds to a straight molecule, which by definition is not biaxial, and indeed in this case $\cos \theta=-1$ and $\lambda=0$. The special value of $\lambda=\frac{1}{\sqrt{6}}$ (see e.g. Fig 2 below, and discussed extensively below [18, 19]), at which biaxiality is maximal, corresponds to the tetrahedral angle $\theta=$ $\cos ^{-1}(-1 / 3) \approx 109.47^{\circ} \approx 0.61 \pi$.

Eq.(26) can be inverted so that for a liquid crystal whose biaxiality can be ascribed to bent-core effects alone, the bent-core angle can be expressed in terms of the biaxiality parameter:

$$
\cos \theta=-\left(\frac{1-\sqrt{\frac{2}{3}} \lambda}{1+\sqrt{6} \lambda}\right)
$$

\section{B. Zero-field phase diagram}

We first recall the influence of the molecular biaxiality on the isotropic-uniaxial nematic transition temperature within the geometric-mean approximation. The phase diagram as a function of temperature and degree of biaxiality (see Fig.2) was derived by Boccara et al.[18] and later by Remler and Haymet [19]. These studies also included the biaxial nematic phase, which we do not discuss here.

Fig.2 shows the dependence of the scaled transition temperature, $T_{N I}^{*}$, on the relative biaxiality parameter $\lambda$. The key features of this phase diagram are as follows.

(a) The phase transition temperature $T_{N I}^{*}$ is an increasing function of the biaxiality $\lambda$.

(b) At a critical value of the biaxiality $\lambda=\lambda_{m}=\frac{1}{\sqrt{6}}$, the continuous $N_{U}^{+}-N_{B}$ transition collides with the first order $I-N_{U}^{+}$transition at what is known as 


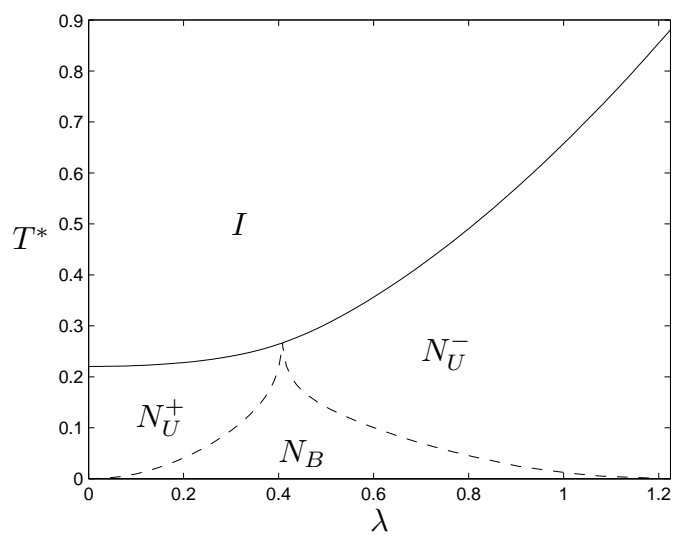

FIG. 2. Liquid crystal phase as a function of scaled temperature $T^{*}$ and biaxiality $\lambda$, within the GMA, as calculated e.g. in ref. [18]. Continuous lines: first-order transitions. Broken lines: continuous transitions. $I$ : isotropic phase. $N_{U}^{+}$: uniaxial nematic phase formed from calamitic molecules. $N_{U}^{-}$: uniaxial nematic phase formed from discotic molecules. $N_{B}$ : biaxial nematic phase.

the Landau point. This is a continuous multicritical point, at which there is a direct $I-N_{B}$ transition without an intermediate uniaxial phase.

(c) As $\lambda$ is increased beyond $\lambda_{m}$, the molecular ordering axis switches, as the major and minor symmetry axes exchange roles. The phase diagram possesses some reflection symmetry around $\lambda_{m}$ (discussed in more detail, e.g in $[31,32])$, so that the phase $N_{U}^{+}$ and $N_{U}^{-}$can be regarded as alternative representations of the same phase. Equivalently, for each $\lambda>\lambda_{m}$ it is possible to redefine axes in such a way as to associate this value of $\lambda$ with a lower degree of biaxiality $\lambda^{\prime}$.

Thus the value $\lambda_{m}$ may be regarded as corresponding to a maximal degree of biaxiality. A comprehensive study of the effects of biaxiality thus only requires a study of values of $\lambda \leq \lambda_{m}$, and this is the procedure which we adopt.

\section{Method}

Rather than solve eq.(18) directly, we minimise the Helmholtz free energy

$$
A^{*}=\frac{A}{u_{200}}=-T^{*} \ln Q\left(\mathcal{J}, T^{*}\right)+\frac{\mathcal{J}^{2}}{2},
$$

with $Q(\mathcal{J})$ defined as in eq.(19). The first derivative of this equation is the self-consistent equation eq.(18). We note that there are some subtle analytic points, which are addressed, for example by Katriel et al.[16]. Strictly speaking this procedure is not valid everywhere for finding minimisers of $A[f(\omega)]$. However, it is valid for finding stationary points. In the region of interest the method also suffices for determining the present quantities of interest.

The procedure determines the equilibrium order parameter $\mathcal{J}$ at a given temperature. The minimisation uses the MATLAB function fmincon and iterates toward a solution using a quasi-Newton method [33]. The nematic-to-paranematic phase transition temperature is found by determining $\mathcal{J}$, as a function of $T^{*}, V, \lambda$. The first-order transition at $T_{N P}$ is located when there is a discontinuous change in the order parameter $\mathcal{J}$ as a function of $T^{*}$.

\section{Results}

We first give a general overview of the biaxiality effects. In Figs. 3 we show the dependence of the order parameter $\mathcal{J}$ on the scaled temperature for a number of different values of external field $V$ and biaxiality $\lambda$. In each subfigure, biaxiality is constant. For zero field strength, the first-order transition is between the uniaxial nematic and the isotropic phases. As $\lambda$ is increased, the zero-field transition temperature $T_{N P}$ increases. This result is already known [18, 26]. However, in some sense, in the context of this paper, this result is less interesting, for in any given case, the overall magnitude of the orienting forces will not be known. Rather, it is the fine structure of the phase diagram close to the onset of nematic behaviour, with or without an imposed external field, which is of interest. As field $V$ increases, the order parameter jump $\Delta \mathcal{J}$ becomes smaller, reducing to zero at a critical point at $\left(V_{c}, T_{c}^{*}\right)$. For $V>V_{c}$, the nematic phase and the paranematic phase are no longer distinguishable.

As $\lambda$ increases, the difference between the zero-field transition temperature, $T_{N I}^{*}$, and the critical transition temperature, $T_{c}^{*}$, (at the critical field) becomes smaller. This phenomenon is related to the fact that $\mathcal{J}$ at the phase transition in zero field becomes smaller as $\lambda$ increases. We note that this feature is likely to be robust against changes in the overall magnitude of the orienting force. Higher degrees of biaxiality are expected to show lower order parameters at the phase transition.

Our calculations also illuminate the effect of biaxiality on the Cotton-Mouton parameter, as discussed above in eqs.(24,25). Evaluation of eq.(23) for specific $\lambda$ yields:

$$
T_{b f}^{*}=\frac{1+2 \lambda^{2}}{5}
$$

and hence a Cotton-Mouton parameter

$$
\kappa=\frac{\left(2 \lambda^{2}+1\right)}{5\left(T^{*}-T_{b f}^{*}\right)} .
$$

The maximum value of the Cotton-Mouton coefficient 


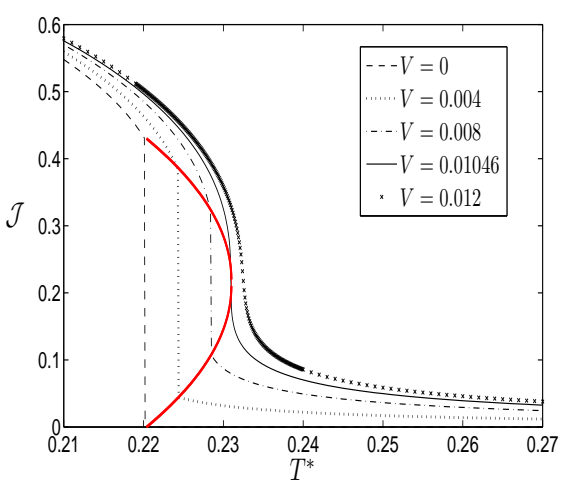

(a) $\lambda=0$

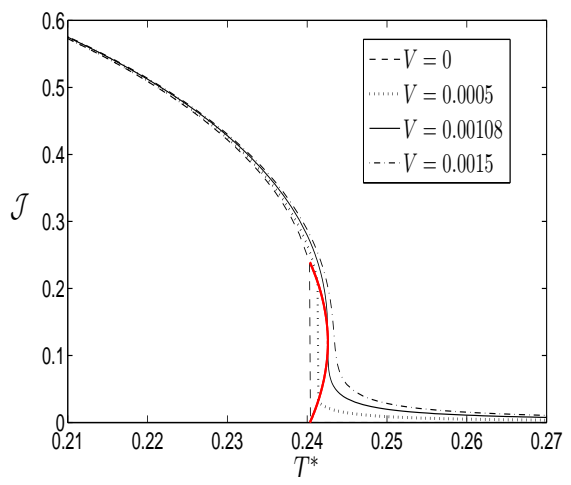

(c) $\lambda=0.3$

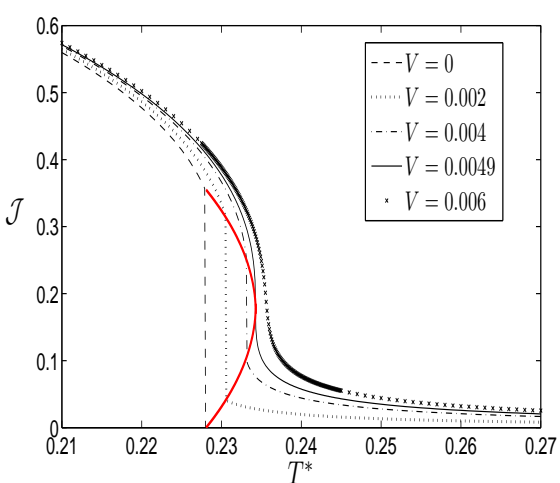

(b) $\lambda=0.2$

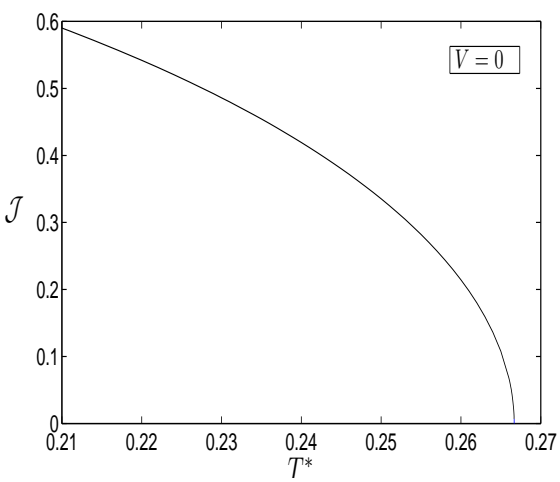

(d) $\lambda=1 / \sqrt{6}$

FIG. 3. Dependence of the composite orientational order parameter $\mathcal{J}$ on scaled temperature $T^{*}$, for different field strengths $V$, for several different molecular biaxiality parameters $\lambda$, varying from $\lambda=0$ (uniaxial molecules) to $\lambda=1 / \sqrt{6}$ (maximal biaxiality). For maximal biaxiality, all non-zero constant $V$, all curves (not shown) would be rounded. Nematic-paranematic coexistence curves shown in bold (red online).

occcurs at $\kappa_{\max }=\frac{\left(2 \lambda^{2}+1\right)}{5\left(T_{p n}^{*}-T_{b f}^{*}\right)}$. As the degree of biaxiality increases, even at zero field, the denominator of this quantity tends to zero, and so $\kappa_{\max } \rightarrow \infty$ as $\lambda \rightarrow \frac{1}{\sqrt{6}}$. As expected, a susceptibility diverges at the biaxial critical point.

We now pass to the dependence of the scaled transition temperature on the scaled effective field $V$ for different degrees of biaxiality $\lambda$, shown in Fig.4. In each case, phase transition curve appears to be linear. There are two limiting cases. The first is $\lambda=0$, the uniaxial case (curve A in Fig.4), which is well-known. The second occurs for $\lambda=\frac{1}{\sqrt{6}}$, at maximal biaxiality. In this case the line shrinks to a point, consistent with the known fact that in this case, the first order phase transition reaches a critical point. Between these limiting cases, the length of the line shrinks steadily as the degree of biaxiality increases. The implication, consistent with remarks made in ref.[19], is that the field required to reach the critical point is dramatically reduced for highly biaxial liquid crystals.

We note also that the slope of the line increases as it shortens. The fact that the length of the magnetic coexistence line reduces with increasing biaxiality is in some sense a consequence of the approach of the nematicisotropic line to the multicritical Landau point. At this point, the magnetic critical point must collide with the phase transition; now the addition of even a small magnetic field destroys the phase transition. We can, however, also ask why these lines appear to be so straight, and why the slope increases with the degree of biaxiality.

We shall return to this point in the next section, where we discuss the Landau-de Gennes theory of the same phenomenon, which is exactly soluble. But it turns out that we can obtain quite detailed understanding of these phenomena using thermodynamic considerations. The shape of a first-order transition line in a general phase space is governed by a generalised Clausius-Clapeyron equation [34]. This relates the shape of the curve to properties which change across the transition. In this case, we find 
that

$$
\frac{\partial T}{\partial V} \sim \frac{\delta S}{\delta \mathcal{S}}
$$

where $\delta S$ is the change in order parameter across the phase transition, and $\delta \mathcal{S}$ is the analogous change in entropy. These considerations enable us to derive a differential equation for the shape of the coexistence curve $T_{p n}(V)$, as follows.

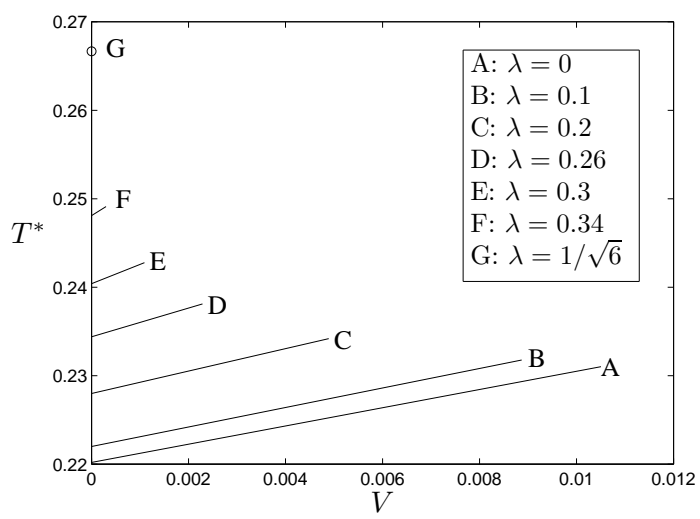

FIG. 4. Dependence of transition temperature on scaled quadratic magnetic flux density, $V$, as molecular biaxiality $\lambda$ increases.

We first recall the Helmholtz free energy in equilibrium, using eqs. $(7,10,12,19,28)$ :

$$
\begin{aligned}
& A^{*}=-T^{*} \ln Q\left(\mathcal{J}, T^{*}, V\right)+\frac{1}{2} \mathcal{J}^{2} \\
& =T^{*} \int d \omega f(\omega) \ln f(\omega)-\frac{1}{2} \mathcal{J}^{2}-V \mathcal{J} .
\end{aligned}
$$

Let us suppose that there is coexistence at $\left(T^{*}, V\right)$, between states defined by $\mathcal{J}_{1}, \mathcal{J}_{2}$, with free energies $A_{1}^{*}\left(\mathcal{J}_{1}\right)=A_{2}^{*}\left(\mathcal{J}_{2}\right)$. Then along the coexistence curve

$$
\frac{d A_{1}^{*}}{d T^{*}} \Delta T^{*}+\frac{d A_{1}^{*}}{d V} \Delta V=\frac{d A_{2}^{*}}{d T^{*}} \Delta T^{*}+\frac{d A_{2}^{*}}{d V} \Delta V,
$$

where $\left(T^{*}+\Delta T^{*}, V+\Delta V\right)$ is also on the coexistence curve. The ratio $\frac{\Delta T^{*}}{\Delta V}$ then defines the differential equation for the coexistence curve in $\left(T^{*}, V\right)$ phase space.

We further note that, in view of the equlibrium condition that $\frac{\partial A^{*}}{\partial \mathcal{J}}=0$, the total derivatives of the free energy are equal to the partial derivatives keeping $\mathcal{J}$ constant. Thus, from the last term of the extreme right hand side of eq.(33) we obtain that

$$
\frac{d A^{*}}{d V}=-\mathcal{J}
$$

Furthermore we can write

$$
A^{*}=\frac{U}{u_{200}}-T^{*} \mathcal{S}=-\frac{1}{2} \mathcal{J}^{2}+\left(-T^{*} \ln Q+\mathcal{J}^{2}\right),
$$

thus isolating an expression for the entropy per particle

$$
\frac{\mathcal{S}}{k_{B}}=-\frac{\partial A^{*}}{\partial T^{*}}=\ln Q-\frac{\mathcal{J}^{2}}{T^{*}} .
$$

Now, combining eqs. $(33,34,35,36)$, we obtain

$$
\begin{array}{r}
\left(-\ln Q_{1}+\ln Q_{2}+\frac{\left(\mathcal{J}_{1}^{2}-\mathcal{J}_{2}^{2}\right)}{T^{*}}\right) \Delta T^{*} \\
\left.=\left(\mathcal{J}_{1}-\mathcal{J}_{2}\right)\right) \Delta V .
\end{array}
$$

This may be further simplied by noting that

$$
A_{1}^{*}=-T^{*} \ln Q_{1}+\frac{1}{2} \mathcal{J}_{1}^{2}=-T^{*} \ln Q_{2}+\frac{1}{2} \mathcal{J}_{2}^{2}=A_{2}^{*},
$$

yielding

$$
-\ln Q_{1}+\ln Q_{2}=-\frac{\left(\mathcal{J}_{1}^{2}-\mathcal{J}_{2}^{2}\right)}{2 T^{*}} .
$$

Combining eqs.(37,39), we obtain

$$
\left(\frac{\left(\mathcal{J}_{1}^{2}-\mathcal{J}_{2}^{2}\right)}{2 T^{*}}\right) \Delta T^{*}=\left(\mathcal{J}_{1}-\mathcal{J}_{2}\right) \Delta V .
$$

Simplifying this further yields the final ClausiusClapeyron relationship for $T^{*}(V)$ along the coexistence curve:

$$
\frac{\Delta T^{*}}{\Delta V}=2 T^{*}\left(\frac{\mathcal{J}_{1}-\mathcal{J}_{2}}{\mathcal{J}_{1}^{2}-\mathcal{J}_{2}^{2}}\right)=\left(\frac{2 T^{*}}{\mathcal{J}_{1}+\mathcal{J}_{2}}\right) .
$$

Eq.(41) has two immediate corollaries. The first concerns the nature of the curve $T^{*}(V)$ for a given value of the biaxiality parameter $\lambda$. In this case the sum $\mathcal{J}_{1}+\mathcal{J}_{2}$ may be regarded as approximately constant, as in $\mathcal{J}$ space, it will be locally symmetrical about the magnetic critical point; then $\mathcal{J}_{1}+\mathcal{J}_{2} \approx 2 \mathcal{J}_{c}$, and $\frac{\Delta T^{*}}{\Delta V} \approx \frac{T_{c}^{*}}{\mathcal{J}_{c}}$, as the temperature also does not change much along this line.

The second corollary is concerned with the slope of the line as the biaxiality parameter is changed. Now $\mathcal{J}_{2}=0$, and $\mathcal{J}_{1}=\mathcal{J}\left(T_{N I}\right)$. In this case, eq.(41) reduces to

$$
\frac{\Delta T^{*}}{\Delta V}=\frac{2 T_{N I}^{*}}{\mathcal{J}\left(T_{N I}, \lambda\right)} .
$$

This is a property only of the zero-field theory [18, 19], in which the quantity $\mathcal{J}(\lambda) \sim\left(\lambda_{m}-\lambda\right)$ as $\lambda \rightarrow \lambda_{m}$. Thus $\frac{\Delta T^{*}}{\Delta V}$ diverges as the Landau multicritical point is approached, as has been observed in the computations.

Finally we examine the phase transition lines in the whole biaxiality-temperature plane. In Fig.5 we show the effect of the molecular biaxiality on the orientational spinodal line $\left(T_{b f}^{*}\right)$, the transition line $\left(T_{N I}^{*}\right)$ and the magnetic critical temperature $\left(T_{c}^{*}\right)$. All three quantities increase with increasing biaxiality, but this cannot be used as a diagnostic tool in interpeting experiments. 
However, the gaps between the three curves decrease monotonically, tending to zero as $\lambda \rightarrow \lambda_{m}$. This feature is more robust than the secular trend in the curves themselves. It seems that smaller values of $T_{N I}^{*}-T_{b f}^{*}$ and of $T_{c}^{*}-T_{N I}^{*}$ are signatures of the effect of biaxiality, although the numbers are, of course, open to question.

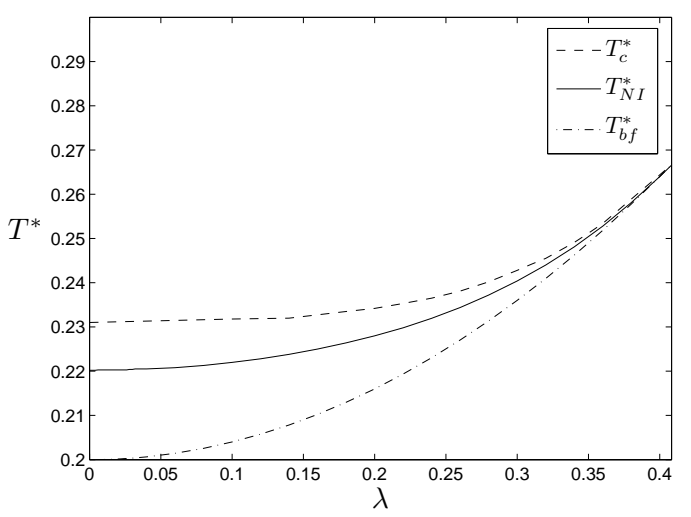

FIG. 5. Dependence of scaled bifurcation temperature $T_{b f}^{*}$, scaled transition temperature $T_{N I}^{*}$ and scaled critical temperature $T_{c}^{*}$ on biaxiality parameter, $\lambda$.

One interesting feature of the results is that within the molecular field theory the ratio

$$
r=\frac{T_{c}^{*}-T_{b f}^{*}}{T_{N P}^{*}-T_{b f}^{*}},
$$

is very nearly constant, whatever the degree of biaxiality, with $r \approx 1.5$, and with the departures from this value always remaining less than $4 \%$. We shall return to this result later in the paper, comparing it with the analytic predictions of Landau-de Gennes theory and also with experiment.

\section{LANDAU-DE GENNES THEORY}

\section{A. General remarks}

In this section we develop a Landau-de Gennes theory [35] to describe magnetic field effects in nematogens composed of biaxial molecules close to the onset of nematic order. The theory is designed to illuminate the molecular field theory developed in the last section, and also specifically to address questions raised by Ostapenko et al. [6] in their recent study of magnetic field effects in bent-core nematics. In general such theories exchange simplicity for a degree of accuracy, but do have the advantage of using as input parameters which are obtained from experiment. In uniaxial nematogens Landau-de Gennes theories have already been used some time ago to describe the effects of magnetic [1, 7, 9] and electric [4, 36] fields. There has also been considerable work on Landau-de Gennes theories in biaxial nematogens in zero field [1, 37-43].
A particular problem in discussing Landau theories of biaxial fluids is the necessity to account for order parameters associated with all molecular axes, rather than merely the single major order parameter associated with ordering of the long axis. This problem can be addressed using a full set of Wigner matrices [19], or a supertensor [37], or by introducing a second ordering tensor associated with ordering of the minor axes [21] (see Turzi [44] for a comparative discussion). Here we adopt this latter approach. In a uniaxial nematic each of these tensors is itself uniaxial, and the order parameter associated with the minor axes is known as the molecular biaxiality order parameter (as opposed to the phase biaxiality order parameter, which only occurs in a biaxial phase). Once orientational degeneracy is eliminated, the two required scalar quantities in the expansion close to the onset of uniaxial nematic order are the parameters $S$ and $D$ defined in eqs.(2). The construction of the complete Landau-de Gennes theory, however, is contingent on the correct enumeration of a menagerie of tensor invariants, with a large number of consequent parameters as coefficients, even if the theory is truncated at a relatively low order. It turns out to be hard to relate changes in these essentially phenomenological coefficients to molecular properties, and thus to gain insight into the relationship between the structure of the critical points and the molecular biaxiality.

Some of the present authors [16, 17] have adopted an intermediate Landau-de Gennes strategy. In this approach, parameters in the Landau theory of a biaxial nematogen have been directly associated with quantities which occur in the molecular field theory. In general, a minimal Landau-de Gennes model close to and in the uniaxial nematic phase still requires an expansion in both $S$ and $D$. However, by employing the geometric mean approximation discussed in section II A above, it turns out to be possible, as in the molecular field theory, to replace the independent use of $S$ and $D$ by the composite order parameter $\mathcal{J}=S+2 \lambda D$, where $\lambda$ is the relative biaxiality parameter used in the previous section.

We shall suppose that the addition of an external field involves a single extra one-particle interaction term in the free energy. This term is associated with the anisotropic interaction between the order parameters and the external field. This is consistent with the approach adopted in section II, but there are limitations to this approximation. In the case of magnetic fields, the intermolecular interactions will not be changed by the application of the field, and hence the simple one-particle anisotropyenergy is valid. But in the case of an electric field, under some circumstances a macroscopic polarization may be introduced, as discussed by Rjumtse et al. [45]; then the interaction between macroscopic dipoles may no longer be negligibly small. 


\section{B. Free energy}

We use the free energy expansion introduced by Luckhurst et al.[17] to obtain the following order parameter expansion, truncated at fourth order:

$$
\begin{aligned}
A^{*}=(3 / 4) a\left(T^{*}-\right. & \left.T_{b f}^{*}\right) \mathcal{J}^{2}-(1 / 4) b T^{*} \mathcal{J}^{3} \\
& +(9 / 16) c T^{*} \mathcal{J}^{4}-V \mathcal{J} .
\end{aligned}
$$

The coefficients in this expansion are now explicitly dependent on the biaxiality parameter $\lambda$, but also dependent on the temperature $T^{*}$ :

$$
\begin{aligned}
& T_{b f}^{*}=\left(1+2 \lambda^{2}\right) / 5, \\
& a=\frac{10}{3\left(1+2 \lambda^{2}\right)}, \\
& b=\left(\frac{100}{21}\right) \frac{\left(1-6 \lambda^{2}\right)}{\left(1+2 \lambda^{2}\right)^{3}}, \\
& c=\left(\frac{100}{441}\right) \frac{\left(56 \lambda^{6}+444 \lambda^{4}-78 \lambda^{2}+17\right)}{\left(1+2 \lambda^{2}\right)^{5}} .
\end{aligned}
$$

The parameter $V$ has the same significance as in eq.(8), and makes the same assumption about the relative size of external field interaction with $S$ and $D$. The spinodal temperature $T_{b f}^{*}(\lambda)=\left(1+2 \lambda^{2}\right) / 5$ is consistent with that derived from the molecular field theory. More importantly the cubic term proportional to $b$ vanishes as $\left(\lambda_{m}-\lambda\right)$ at the Landau point. This forces the transition at the Landau point (and only at that point) to be continuous, and leads to other anomalies in the system as $\lambda \rightarrow \lambda_{m}$.

Expression (45) explicitly ignores order parameters associated with the phase ordering and hence the biaxial nematic phase. As we are not concerned with the biaxial $N_{B}$ phase, this approximation is harmless here, but would be unacceptable if we were also including effects associated with the $N_{B}$ phase. Finally we note that although the fourth order coefficient is a complicated function of $\lambda$, it is a well-behaved complicated function, and thus no thermodynamic anomalies are expected. For further details we refer the reader to ref. [17]

As we shall be evaluating expressions only in the neighbourhood of the nematic-isotropic transition, we adopt the further approximation of forcing third and fourth order coefficients to be constant. We do this by replacing $T^{*}$ in eq.(44) by a constant value $\theta=0.22 \approx T_{N I}(\lambda=0)$. We thus rewrite the Landau-de Gennes free energy as

$$
\begin{aligned}
A^{*}=(3 / 4) a\left(T^{*}-\right. & \left.T_{b f}^{*}\right) \mathcal{J}^{2}-(1 / 4) \mathcal{B J}^{3} \\
& +(9 / 16) \mathcal{C} \mathcal{J}^{4}-V \mathcal{J}
\end{aligned}
$$

with

$$
\begin{aligned}
& T_{b f}^{*}=\left(1+2 \lambda^{2}\right) / 5 \\
& a=\frac{10}{3\left(1+2 \lambda^{2}\right)}, \\
& \mathcal{B}=\left(\frac{100}{21}\right) \frac{\left(1-6 \lambda^{2}\right)}{\left(1+2 \lambda^{2}\right)^{3}} \theta \\
& \mathcal{C}=\left(\frac{100}{441}\right) \frac{\left(56 \lambda^{6}+444 \lambda^{4}-78 \lambda^{2}+17\right)}{\left(1+2 \lambda^{2}\right)^{5}} \theta .
\end{aligned}
$$

The partitioning of factors in the coefficients in eqs. $(46,47)$ are those introduced by Gramsbergen et al.[1], and facilitate comparison with previous work.

\section{Non-dimensionalization}

The Landau-de Gennes magnetic critical point has been discussed by Gramsbergen et al. [1]. Here we rederive these solutions, using scaling properties of this expansion. We make the following rescalings for the order parameter, the temperature, the free energy density and the external field respectively:

$$
\begin{aligned}
\tilde{J} & =\frac{9 \mathcal{C}}{2 \mathcal{B}} \mathcal{J}, \\
t & =\frac{27 a \mathcal{C}}{\mathcal{B}^{2}}\left(T^{*}-T_{b f}^{*}\right) . \\
\tilde{A} & =729 \frac{\mathcal{C}^{3}}{\mathcal{B}^{4}} A^{*} \\
v & =\frac{162 \mathcal{C}^{2}}{\mathcal{B}^{3}} V .
\end{aligned}
$$

The rescaled free energy can now be written in terms of $\tilde{J}, t$ in the following universal form:

$$
\tilde{A}=-v \tilde{J}+t \tilde{J}^{2}-2 \tilde{J}^{3}+\tilde{J}^{4} .
$$

This rescaled free energy is independent of the biaxiality parameter $\lambda$. All critical properties away from the Landau point (where the transformation does not apply) can be derived using eq.(49). Critical properties of $V_{c}(\lambda)$, and $\frac{\Delta T^{*}}{\Delta V}$ along the coexistence curve, as $\lambda \rightarrow \lambda_{m}$ follow from the behaviour of $\mathcal{B}(\lambda)$, i.e. from the scaling transformation, rather than from the intrinsic properties of the model itself.

\section{Properties of non-dimensionalized model}

We shall investigate the properties of the nondimensionalized model using elementary algebraic arguments. We thus avoid the necessity of investigating minima and solving complicated simultaneous equations. However, the mathematical tricks depend crucially on 
truncating the expansion at fourth order. If this condition is relaxed, the argument no longer holds, although some of the results remain approximately true.

The zero-field spinodal $T_{b f}^{*}$ corresponds to $t=0$, and the $N I$ transition temperature $T_{N I}^{*}$ occurs at $t=1$, at which point the free energy can be written as:

$$
\tilde{A}=\tilde{J}^{2}(\tilde{J}-1)^{2} .
$$

This has equilibria at $\tilde{J}=0,1$ with $\tilde{A}=0$, thus exhibiting explicitly that at the zero-field transition the rescaled order parameter is unity.

Along the NP phase transition line, let the order parameter take values $\tilde{J}_{1}, \tilde{J}_{2}$ in the $N, P$ phase respectively. $\tilde{A}(\tilde{J})$ is quartic in $\tilde{J}$, with equal minima at $\tilde{J}_{1}, \tilde{J}_{2}$, and must necessarily take the form

$$
\tilde{A}_{N P}=\zeta+\left(\tilde{J}-\tilde{J}_{1}\right)^{2}\left(\tilde{J}-\tilde{J}_{2}\right)^{2},
$$

where $\zeta(v)$ varies along the phase transition line, the unit factor outside the bracketed term is implied by the unit coefficient of the quartic term, the repeated factors imply the minima (and hence equilibria) at $\tilde{J}_{1}, \tilde{J}_{2}$, and the explicit form implies the equality of the minima at $\zeta$. Expanding eq.(51) explicitly, we obtain

$$
\begin{array}{r}
\tilde{A}=\left[\zeta+\tilde{J}_{1}^{2} \tilde{J}_{2}^{2}\right]-2 \tilde{J}_{1} \tilde{J}_{2}\left[\tilde{J}_{1}+\tilde{J}_{2}\right] \tilde{J} \\
+\left[\tilde{J}_{1}^{2}+\tilde{J}_{2}^{2}+4 \tilde{J}_{1} \tilde{J}_{2}\right] \tilde{J}^{2}-2\left[\tilde{J}_{1}+\tilde{J}_{2}\right] \tilde{J}^{3}+\tilde{J}^{4}
\end{array}
$$

which in principle is identical with eq.(49).

Further progress can be made by comparing coefficients respectively of terms of cubic, quadratic and linear order in eqs. $(49,52)$, yielding the relationships:

$$
\begin{aligned}
1 & =\tilde{J}_{1}+\tilde{J}_{2}, \\
t & =\tilde{J}_{1}{ }^{2}+\tilde{J}_{2}{ }^{2}+4 \tilde{J}_{1} \tilde{J}_{2}=1+2 \tilde{J}_{1} \tilde{J}_{2}, \\
v & =2 \tilde{J}_{1} \tilde{J}_{2}\left(\tilde{J}_{1}+\tilde{J}_{2}\right)=2 \tilde{J}_{1} \tilde{J}_{2} .
\end{aligned}
$$

Eq.(53a) shows that the approximate relation discussed in eq.(41) that the constancy of the sum of the order parameters across the transition line along its length is rigorously true in the Landau-de Gennes theory. Combining eqs. $(53 \mathrm{~b}, 53 \mathrm{c})$ and eliminating $\tilde{J}_{1} \tilde{J}_{2}$ yields a universal explicit equation for the magnetic coexistence line:

$$
t=1+v \Rightarrow \frac{\Delta t}{\Delta v}=1
$$

The critical point $v_{c}$ occurs when the $N$ and $P$ phases collide, i.e. $\tilde{J}_{1}=\tilde{J}_{2}=\frac{1}{2}$, and hence using eq.(53c), we find that

$$
v_{c}=\frac{1}{2} \text {. }
$$

Combining this with eq.(54) yields

$$
t_{c}=1+v_{c}=\frac{3}{2} .
$$

Finally we derive relations for the order parameter difference across the transition line. Using eqs.(53a,53c,55a), we derive

$$
\begin{aligned}
\left(\tilde{J}_{1}-\tilde{J}_{2}\right)^{2} & \equiv\left(\tilde{J}_{1}+\tilde{J}_{2}\right)^{2}-4 \tilde{J}_{1} \tilde{J}_{2} \\
& =1-2 v=2\left(v_{c}-v\right),
\end{aligned}
$$

and likewise, using eq.(54):

$$
\left(\tilde{J}_{1}-\tilde{J}_{2}\right)^{2}=2\left(t_{c}-t_{N P}\right) \text {. }
$$

\section{E. Results}

We now use results from the last subsection to derive general conclusions. Result eq.(55b) that $t_{c}=\frac{3}{2}$ translates, when rescaled into physical units to

$$
r_{L d G}=\frac{T_{c}^{*}-T_{b f}^{*}}{T_{N I}^{*}-T_{b f}^{*}} \equiv 1.5,
$$

an analytic exact result which agrees with the numerically calculated value of $r$ from the molecular field theory in eq. (43). The value of $\left(T_{c}^{*}-T_{b f}^{*}\right)$, on the other hand, depends on the value of $\lambda$, and behaves as $\left(\lambda_{m}-\lambda\right)^{2}$ as $\lambda \rightarrow \lambda_{m}$.

The value of the critical field itself, from eqs.(48,55a) is given by:

$$
V_{c}=\frac{\mathcal{B}^{3}}{324 \mathcal{C}^{2}}
$$

from eq.(45), this predicts that $V_{c} \sim\left(\lambda_{m}-\lambda\right)^{3}$ and the critical magnetic field $B_{c} \sim\left(\lambda_{m}-\lambda\right)^{3 / 2}$.

Using eq.(54), together with eq.(48), we obtain the slope of the $N P$ coexistence curve:

$$
\frac{\Delta T_{N P}^{*}}{\Delta V}=\frac{6 \mathcal{C}}{a \mathcal{B}}=\frac{4}{3 a \mathcal{J}_{N I}},
$$

noting the same $\mathcal{J}^{-1}$ dependence as in the molecular field case eq.(42).

Using eqs.(47), this can be expressed in terms of the biaxiality parameter as

$$
\frac{\Delta T_{N P}^{*}}{\Delta V}=\frac{f(\lambda)}{\lambda_{m}-\lambda},
$$

where

$$
f(\lambda)=\frac{56 \lambda^{6}+444 \lambda^{4}-78 \lambda^{2}+17}{70\left(1+2 \lambda^{2}\right)\left(\lambda_{m}+\lambda\right)} .
$$

We can also rescale the equation for the coexistence curve in $\left(\mathcal{J}, T^{*}\right)$ coordinates eq. $(56 \mathrm{~b})$ to obtain

$$
\left(\mathcal{J}_{1}-\mathcal{J}_{2}\right)^{2}=\frac{8 a}{3 \mathcal{C}}\left(T_{c}^{*}-T_{N P}^{*}\right)
$$

In the purely uniaxial case $\lambda=0$, this reduces, using eq.(45), to

$$
\left(\mathcal{J}_{1}-\mathcal{J}_{2}\right)^{2}=\frac{196}{85 \theta}\left(T_{c}^{*}-T_{N P}^{*}\right) .
$$




\section{F. Comparison between Landau-de Gennes and molecular field theories}

Since our Landau-de Gennes theory is derived from the molecular field theory, it is not surprising that both theories have the same qualitative predictions. Although in principle the molecular field theory is more accurate, the Landau-KKLS theory has the advantage that it is analytically accessible. In fact in many cases the theories agree even semi-quantitatively. Key common predictions are that $T_{N P}$ increases quadratically with the applied field strength and that the rate of change of the transition temperature with $B^{2}$ increases as the molecular biaxiality becomes larger, as shown for the molecular field theory in eq.(42) and for the Landau-deGennes theory in eq. (59). This increase in the rate of change of $T_{N P}$ for the two theories is shown in Fig.6. We can see that the difference in the rate of change for calamitic molecules and for bent-core molecules is significant, particularly close to the Landau point. The temperature scale over which the magnetic coexistence line occurs is predicted to be of the same order of magnitude as $T_{N I}-T_{b f}$. For mesogenic systems of higher molecular biaxiality, a lower magnetic field strength is required to observed the magnetic critical point.

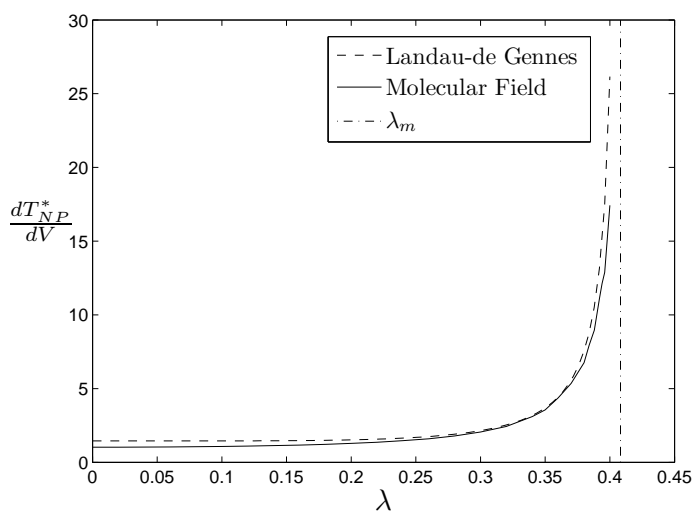

FIG. 6. Dependence of rate of change of $d T_{N P}^{*} / d V$ with molecular relative biaxiality $\lambda$.

\section{DISCUSSION AND CONCLUSIONS}

\section{A. Comparison with experiment}

This study was prompted by an experiment by Ostapenko et al. [6] which found a significant increase in the transition temperature due to a magnetic field for a system of bent-core molecules. These molecules are often highly biaxial. We have developed a molecular field theory to study the effect of the molecular biaxiality on the magnetic field-induced nematics, and calculated the consequence of this theory. We have also extended to finite fields previous work in which we developed the analogous Landau-de Gennes free energy for a biaxial liquid crystal of given molecular biaxiality [17]. We note that an earlier study [5] by the same research group found that $T_{N I}-T_{b f} \approx 0.4 K$ was anomalously small. This study provides extra indirect calorimetric evidence of the biaxiality of the bent-core molecules used in the experiments of Ostapenko et al. [6], for both our work and that of others $[18,19]$ also predict reduced values of $T_{N I}-T_{b f}$. In addition, other techniques provide more direct evidence of these biaxial effects [46].

The background to the experimental studies is that it has turned out to be very difficult to persuade nematogens to exhibit a magnetic-field-dependent transition $[2,3]$, and that when it was observed it was extremely weak. Thus the unusual experimental observation was simply that for this system it was possible to observe a noticeable magnetic-field dependence of the nematic-isotropic phase transition. Electric field effects are in principle analogous from a theretical point of view, and should be easier to observe, because the electric coupling to experimentally accessible electric field is higher than that to experimentally accessible magnetic fields $[4,36,45]$. But electric fields present other kinds of problems, most noticeably Ohmic heating and screening, and so in some sense a magnetic field experiment presents a more unambiguous test of theory.

We remind readers that we have denoted the shift in the nematic-isotropic transition as a temperature dependence of $T_{N P}$, the nematic-paranematic phase transition. Once a field is imposed, the high temperature phase is slightly ordered and is no longer isotropic; conventionally this is now known as a paranematic phase. There is no longer a symmetry change at the phase transition, merely a change in the magnitude of the orientational order parameter. Experimental quantities which have been observed are $\left(T_{N I}-T_{b f}\right) \sim \mathcal{B}^{2} \sim\left(\lambda_{m}-\lambda\right)^{2}[5]$, and $\frac{\Delta T_{N P}}{\Delta B^{2}} \sim \mathcal{B}^{-1} \sim\left(\lambda_{m}-\lambda\right)^{-1}[6]$. The first is historically smaller than in the same quantity measured in calamitics by a factor of about 4.5. The second is dramatically larger than that observed for calamitics [3]. While recent observations with higher fields are not available, and one might suppose that the size of $\left(T_{N I}-T_{b f}\right)$ would be smaller if a whole new set of observations were to be made, the theory is at least consistent with the idea that both these effects are due to the biaxiality-induced proximity of the system to a Landau multicritical point. In general, we expect that if further measurements could be made, the order parameter in the nematic phase at $T_{N I} \sim\left(\lambda_{m}-\lambda\right)$ would be low, and the critical magnetic field $B_{c} \sim\left(\lambda_{m}-\lambda\right)^{3 / 2}$, above which the $P$ and $N$ phases are identical, would also be low compared to uniaxial calamitics.

We can make specific contact with the experimental results of Wiant et al.[5] and Ostapenko et al. [6] by 
using the ratio $r$ defined in eqs. $(43,57)$ :

$$
r=\frac{T_{c}^{*}-T_{b f}^{*}}{T_{N I}^{*}-T_{b f}^{*}}=\frac{T_{c}-T_{b f}}{T_{N I}-T_{b f}} .
$$

This quantity measures the ratio of the length of the $N P$ coexistence curve as a function of temperature with respect to the only temperature scale available, the difference $\left(T_{N I}-T_{b f}\right)$. In both our calculations, we find that $r=1.5$ very closely. But in the experiments [6], it is found that the ratio $r \geq 2.4$, while the critical field has not been reached. The fact that the critical field is not reached is a signature that our goal of measuring $B_{c}$ for a range of calamitics is far-fetched. One does not know how much larger $B_{c}$ is than $B_{\max }$, the largest observed field. On the one hand the closeness of the limit on $r$ to 1.5 is reassuring. On the other hand, the value of $r=1.5$ would seem to be a robust result.

The source for this small but unambiguous experimental disagreement with theory is unclear. It may be that our version of molecular field theory is inaccurate. For example Zhang et al.[47] have performed a cluster calculation involving a more complex self-consistency. They find that $\left(T_{N I}-T_{b f}\right)$ is reduced as compared to the naive molecular field theory. It could be that the estimates of $T_{b f}$ have been made badly, because fluctuation effects are causing the Cotton-Mouton parameter no longer to follow $\left(T-T_{b f}\right)^{-1}$ behaviour. In this context, we remark that the fine structure of the nematic-isotropic transition has long been a puzzle; unreasonably critical in some respects, and unreasonably first-order in others. Alternatively, it could be that other order parameters are playing a role; Ostapenko et al. [6] themselves attributed it to possible tetrahedric order in their system. Nevertheless we can make contact with experiment using the expected biaxiality dependence of the quantity $Z(\lambda)=\frac{T_{N I}^{*}}{T_{b f}^{*}}-1$, assuming that only the biaxiality and nothing else is changing. This quantity is plotted in Fig.7. The quantity $Z(\lambda)$ is a non-dimensionalised measure of how close the nematic-isotropic phase transition is to being a continuous transition. When $Z(\lambda)=0$, the phase transition is continuous.

Wiant et al. $[5]$ measured $T_{N I}-T_{b f}=0.38 K$ for the bent-core LC, 4-chloro-1,3-phenylenebis[4-(4- 9decenyloxy) benzoyloxy benzoate] (10CPBB), yielding a value of $Z \approx 0.001$. We can compare this value to $Z(\lambda=$ $0) \approx 0.0045$. This can be evaluated from computer simulations on the Lebwohl-Lasher model [48], in which the molecules are known to be strictly uniaxial by construction. Alternatively, studies of $5 C B$ and $6 C B$ by Zink and de Jeu [49] yield almost exactly the same answer. If we suppose that the reduction in the quantity $Z$ can be ascribed only to biaxiality, then $Z(\lambda) / Z(0) \approx 0.23$, and we can use Fig. 7 to estimate $\lambda \approx 0.28$. We can now invert result eq. $(27)$ to estimate $\theta(\lambda) \approx 116^{\circ}$, which is rather close to the $120^{\circ}$ expected on chemical grounds for these molecules. If the same calculation is made for the material used in ref.[6], then a value of $\theta \approx 119.5^{\circ}$ is

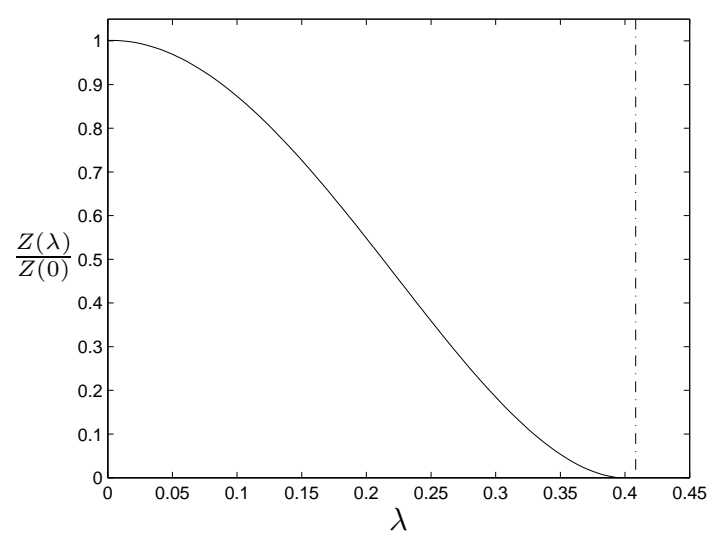

FIG. 7. Dependence of $Z(\lambda)=\left(T_{N I}-T_{b f}\right) / T_{b f}$ as a function of biaxiality parameter, using molecular field theory. Dotted line corresponds to $\lambda=1 / \sqrt{6} \approx 0.41$, the maximal biaxiality.

obtained. This is now even closer to the expected value of $120^{\circ}$, although the extremely close agreement may be fortuitous, as the assumption of a rigid structure does not hold.

Analogous calculations can now be made to predict values of $B_{c}$ for these systems, using the calculated value of $\lambda_{\text {eff }}$. Now, however, the actual value of this depends on the coupling constant, $\chi_{20}$ in eq.(5); one needs to suppose that this does not change, and in addition one needs to estimate $B_{c}$ for a material with zero biaxiality. For the materials discussed in [6], typical values around $30 T$ can be estimated. We note that in this context the estimate for $B_{c}$ in uniaxial systems of $\sim 1000 T$ by Wojtowicz and Sheng [9] was adjusted by Hornreich [7] to $\sim 87 T$; under these circumstances the biaxiality brings down the estimated critical field by a factor of 2 or 3 . We must judge Ostapenko et al.[6] rather unlucky not to have observed the critical point at $31 T$.

\section{B. Discussion of the theory}

Our theory relies on a number of idealizations, whose validity requires some justification. First, we have supposed that molecular field ideas will work, even though the problems under consideration deal with systems in some sense close to critical points, and hence susceptible to the effects of fluctuations. Second, we use the so-called geometric mean approximation (GMA) to reduce an essentially two order parameter problem to that of a single composite order parameter. Quantitatively, this ansatz must work not only for the effective two-particle energy, but also (with the same parameter) for the interaction between a single particle and the external magnetic field. Third, we are supposing that a system of molecules with $C_{2 v}$ symmetry behaves as though it is composed of $D_{2 h}$ symmetry. The idea is that any specifically polar effects associated with the bent-cores do not play a crucial role. 
Molecular field theories within the GMA all predict that the addition of biaxiality will increase the degree of criticality at the nematic-isotropic phase transition, as a Landau multicritical point is approached. At the Landau point a direct transition to a biaxial nematic phase is predicted, but we have concentrated on uniaxial nematic effects (albeit associated with biaxial molecules). The molecular-field approximation is known to grossly overestimate some first-order effects at the phase transition (e.g. the size of orientationally ordered clusters in the disordered phase), while describing correctly others (e.g. the orientational order parameter at the phase transition). We proceed on the assumption that the molecularfield procedure is essentially valid, but that some temperature scales (most precisely $T_{N I}-T_{b f}$ ) are shrunk as compared to the classic molecular field theory. In this case, the Landau- de Gennes theory might be expected to give a reasonable first approximation to a description of the experiments. In any event, there are no other available analytic techniques.

The use of the GMA is predicated on the belief that the underlying interactions are governed either by shape or by dispersion interactions. In these limiting cases, the GMA is known to hold. If the assumptions are relaxed, then the GMA may no longer hold, but the degree to which it is false is not well-studied. Theoretical considerations, however, indicate that relaxation of the GMA may change the phase diagram shown in Fig.2 dramatically. In some cases the Landau multicritical point is destroyed entirely, and replaced by a first-order transition from the isotropic phase to a biaxial nematic [21]. The validity of the GMA ansatz for the interparticle potential is not at all clear in all cases. But a heuristic justification can be constructed, and furthermore, there is an experimental clue. This is precisely that some properties of the system seem more critical (in the phase transition sense) than is usual in nematogenic materials, and hence the system may be close to the Landau multicritical point. So we proceed supposing that at least the Landau multicritical point feature to the theory is retained.

The use of the GMA for the external potential, eq.(6), is less well-justified theoretically. Our main justification lies in terms of providing an easily tractable theory. This form for the order parameter-field coupling seems to compel the magnetic critical point to approach the Landau multicritical point at $\lambda=\lambda_{m}=\sqrt{\frac{1}{6}}$. But Vause [39] suggests a quite complex phase diagram topology connecting the magnetic critical point and the Landau multicritical point. However, Vause [39] implicitly relaxes this approximation, pointing out that beyond $\lambda_{m}$, the principal order parameter will now be perpendicular to the magnetic field, something explicitly forbidden by the form of the coupling adopted in eq.(6). At the moment, the precise degree of robustness of the phase topology we derive is unclear and requires further theoretical work.

Finally, there is the question of the quasi-polar nature of the bent-core molecules. Ryumtse et al.[45] have discussed what might occur (albeit in an essentially uniax- ial molecule) when there are strong electric polar effects. But in this respect magnetic and electric moments are different, because induced electric dipole moments may change the intermolecular interaction. By constrast, only magnetic fields of astrophysical magnitude could have this effect, and by that stage no doubt other effects would already have intervened!

\section{Final remarks}

Our most important results are that high biaxiality, or more specifically proximity to the Landau multicritical point, implies that $T_{N I}$ and $T_{b f}$ (sometimes known as $T^{*}$ in the literature) will be closer together. The implied degree of biaxiality can be inferred from the size of this quantity, and this can be converted into an imputed inter-arm angle in the bent-core molecule. This imputed angle seems to be in semi-quantitative agreement with experiment. In addition, in the presence of biaxiality (and thus in bent-core nematics) $T_{N P}$ will be more sensitive to magnetic field, it will be quadratic in the field, and that the sensitivity will diverge at the Landau multicritical point. The Landau-de Gennes theory, together with the experiments $[5,6]$ gives some insight into how this will occur. The theoretical parameter which responds most obviously to the biaxiality is the cubic term in the Landau-de Gennes theory, $\mathcal{B} \sim\left(\lambda_{m}-\lambda\right)$. Ostapenko et al. [6] understood well that their result followed from anomalously low $\mathcal{B}$. In this paper we have provided some extra justification at a molecular level for their observation, and shown that the behaviour of the bent-core nematics is largely a result of the biaxiality implied by the bent-core structure.

Nevertheless, we emphasise that further work is required. If bent-core molecules with inter-arm angles close to the tetrahedral angle $\theta \approx 109.5^{\circ}$ could be synthesised, it is likely that weaker first-order nematic-isotropic transitions with consequent lower critical magnetic fields could be synthesised. This might finally enable the magnetic critical point to be observed. Alternatively, if the nematic-paranematic transition could be observed using calorimetric methods, rather than (as in refs $[5,6]$ ) by observing turbidity, then it might be possible to extrapolate toward the critical point (and hence locate it accurately) without actually producing the necessary high fields.

From a theoretical point of view, the rather hybrid nature of the present theory implicitly supposes that the weak first-order effects are essentially a superposition of fluctuation effects (weakening the Landau-de Gennes cubic term $\mathcal{B}$ ) with biaxial effects. The latter are understood and are described here, but the former have presented a puzzle ever since the Maier-Saupe theory was formulated in the 1950s. More detailed simulations, in which the biaxiality is well-characterized, may enable the GMA to be tested for molecules of different types of shape. We note simulations by Peroukidis et al. [50] which concentrate specifically on bent-core molecules, 
and by Ghoshal et al.[51] which concentrate on board-like molecules. More detailed lattice simulations, in which the GMA is explicitly violated in model potentials, would expose the consequences of violating our key assumptions, but here the extreme sensitivity of the systems close to the critical point might require sophisticated finite-size scaling techniques [52] In this respect, the comments of Vause [39] about phase diagram topologies close to a Landau multicritical point also require further examination, as do the comments of Ghoshal et al.[53] concerning the interaction between transverse dipoles and biaxial molec-

[1] E. F. Gramsbergen, L. Longa, and W. H. de Jeu, Phys. Rep. 135, 195 (1986).

[2] T. W. Stinson and J. D. Litster, Phys. Rev. Lett. 25, 503 (1970).

[3] C. Rosenblatt, Phys. Rev. A 24, 2236 (1981).

[4] I. Lelidis and G. Durand, Phys. Rev. E 48, 3822 (1993).

[5] D. Wiant, S. Stojadinovic, K. Neupane, S. Sharma, K. Fodor-Csorba, A. Jákli, J. T. Gleeson, and S. Sprunt, Phys. Rev. E 73, 030703 (2006).

[6] T. Ostapenko, D. B. Wiant, S. N. Sprunt, A. Jákli, and J. Gleeson, Phys. Rev. Lett. 101, 247801 (2008).

[7] R. M. Hornreich, Phys. Lett. 109A, 232 (1985).

[8] J. Hanus, Phys. Rev. 178, 737 (1969).

[9] P. J. Wojtowicz and P. Sheng, Phys. Lett. 48A, 235 (1974).

[10] P. Palffy-Muhoray and D. A. Dunmur, Mol. Cryst. Liq. Cryst. 97, 337 (1983).

[11] G. R. Luckhurst and P. Simpson, Chem. Phys. Lett. 95, 149 (1983).

[12] P. Lebwohl and G. Lasher, Phys. Rev. A 6, 426 (1972).

[13] R. Zwanzig, J. Chem. Phys. 39, 1714 (1963).

[14] Warsono, K. Abraha, Y. Yusuf, and P. Nurwantoro, Int. Jour. Bas. Appl. Sci. 10, 19 (2010).

[15] G. R. Luckhurst, C. Zannoni, P. L. Nordio, and U. Segre, Mol. Phys. 30, 1345 (1975).

[16] J. Katriel, G. F. Kventsel, G. R. Luckhurst, and T. J. Sluckin, Liq. Cryst. 1, 337 (1986).

[17] G. R. Luckhurst, S. Naemura, T. Sluckin, K. Thomas, and S. S. Turzi, Phys. Rev. E 85, 031705 (2012).

[18] N. Boccara, R. Mejdani, and L. de Sèze, J. Phys. (France) 7, 149 (1977).

[19] D. K. Remler and A. D. J. Haymet, J. Phys. Chem. 90, 5426 (1986).

[20] G. R. Luckhurst, in Physical Properties of Liquid Crystals Nematics, edited by D. A. Dunmur, A. Fukuda, and G. R. Luckhurst (INSPEC, 2001).

[21] A. M. Sonnet, E. G. Virga, and G. E. Durand, Phys. Rev. E 67, 061701 (2003).

[22] R. Rosso, Liquid Crystals 34, 737 (2007).

[23] A. J. Stone, in The Molecular Physics of Liquid Crystals, edited by G. R. Luckhurst and G. W. Gray (Academic Press, 1979).

[24] A. Sonnet, E. G. Virga, and G. E. Durand, Phys. Rev. E 67, 061701 (2003).

[25] P. G. de Gennes, The Physics of Liquid Crystals (Oxford ular shape.

\section{ACKNOWLEDGMENTS}

TBTT acknowledges financial support of a School Ph.D. Studentship from the School of Mathematical Sciences, University of Southampton. TJS thanks P.I.C. Teixeira and T. Ostapenko for helpful discussions. TJS and TBTT thank the Isaac Newton Institute for Mathematical Sciences, University of Cambridge, for hospitality while some of the final parts of this reseach were carried out.
University Press, Oxford, 1990).

[26] G. R. Luckhurst, in The Molecular Physics of Liquid Crystals, edited by G. R. Luckhurst and G. W. Gray (Academic Press, 1979).

[27] B. Mulder, Phys. Rev. A 39, 360 (1988).

[28] A. Ferrarini, G. R. Luckhurst, P. L. Nordio, and S. J. Roskilly, Liq. Cryst. 21, 373 (1996).

[29] G. R. Luckhurst, Thin Solid Films 393, 40 (2001).

[30] M. A. Bates and G. R. Luckhurst, Phys. Rev. E 72, 051702 (2005).

[31] G. De Matteis, F. Bisi, and E. G. Virga, Continuum Mech. Thermodyn. 19, 1 (2007).

[32] S. S. Turzi and T. J. Sluckin, SIAM J. App. Math 73, 1139 (2013).

[33] C. G. Broyden, J. Inst. Maths Applics 6, 76 (1970).

[34] R. M. Bowley and M. Sánchez, Introductory Statistical Mechanics (Oxford University Press, Oxford, 1999) Chap. 11.

[35] P.-G. de Gennes, Mol. Cryst. Liq. Cryst. 12, 193 (1971).

[36] A. J. Nicastro and P. H. Keyes, Phys. Rev. A 30, 3156 (1984).

[37] D. Allender and M. A. Lee, Mol. Cryst. Liq. Cryst. 110, 331 (1984).

[38] D. Allender, M. A. Lee, and N. Hafiz, Mol. Cryst. Liq. Cryst. 124, 45 (1985).

[39] C. A. Vause, Phys. Lett. 114A, 485 (1986).

[40] M. C. J. M. Vissenberg, S. Stallinga, and G. Vertogen, Phys. Rev. E 55, 4367 (1997).

[41] P. Mukherjee, Liq. Cryst. 24, 519 (1998).

[42] A. E. Prostakov, E. S. Larin, and M. B. Stryukov, Crystallography Reports (Russia) 47, 1041 (2002).

[43] G. De Matteis, A. M. Sonnet, and E. G. Virga, Continuum Mech. Thermodyn. 20, 347 (2008).

[44] S. S. Turzi, J. Math. Phys. 52, 053517 (2011).

[45] E. I. Rjumtse, M. A. Osipov, T. A. Rotinyan, and N. P. Yevlampieva, Liq. Cryst. 18, 87 (1995).

[46] Y. K. Kim, M. Majumdar, B. I. Senyuk, L. Tortora, J. Seltmann, et al., Soft Matter 8, 8880 (2012).

[47] Z. D. Zhang, D. X. Zhang, and Y. B. Sun, Chin. Phys. Lett. 17, 749 (2000).

[48] U. Fabbri and C. Zannoni, Molec. Phys. 58, 763 (1986).

[49] H. Zink and W. H. de Jeu, Mol. Cryst. Liq. Cryst. 124, 287 (1985).

[50] S. Peroukidis, A. G. Vanakaras, and D. J. Photinos, Phys. Rev. E 84, 010702R (2011). 
[51] N. Ghoshal, K. Mukhopadhyay, and S. K. Roy, arxiv:1212.3972[cond-mat-soft] (2013).

[52] Z. Zhang, O. G. Mouritsen, and M. J. Zuckermann, Phys. Rev. Lett. 69, 2803 (1992).
[53] N. Ghoshal, K. Mukhopadhyay, and S. K. Roy, Liq. Cryst. 39, 1381 (2012). 